\title{
Galactic Substructure and Energetic Neutrinos from the Sun and Earth
}

\author{
Savvas M. Koushiappas ${ }^{1}$ and Marc Kamionkowski ${ }^{2}$ \\ ${ }^{1}$ Department of Physics, Brown University, 182 Hope Street, Providence, Rhode Island 02912, USA* \\ ${ }^{2}$ California Institute of Technology, Mail Code 350-17, Pasadena, California 91125, USA ${ }^{\dagger}$
}

(Received 28 July 2009; published 14 September 2009)

\begin{abstract}
We consider the effects of Galactic substructure on energetic neutrinos from annihilation of weakly interacting massive particles that have been captured by the Sun and Earth. Substructure gives rise to a time-varying capture rate and thus to time variation in the annihilation rate and resulting energeticneutrino flux. However, there may be a time lag between the capture and annihilation rates. The energeticneutrino flux may then be determined by the density of dark matter in the Solar System's past trajectory, rather than the local density. The signature of such an effect may be sought in the ratio of the direct- to indirect-detection rates.
\end{abstract}

Numerous experimental probes have confirmed indirectly the presence of a yet unknown form of gravitationally interacting matter in Galactic halos that contributes roughly $20 \%$ of the total cosmic energy density. It is generally assumed that "dark matter" is in the form of some yet undiscovered elementary particle. Among the plethora of proposed theoretical particle dark-matter candidates, weakly interacting, massive particles are favored because they provide, quite generally, the correct relic abundance and because they may be experimentally accessible in the near future. Weakly interacting massive particles (WIMPs) arise naturally in supersymmetric extensions (SUSY) of the Standard Model [1] as well as in models with universal extra dimensions (UEDs) [2].

The two principal avenues toward dark-matter detection are direct detection (DD) via observation of the recoil of a nucleus, when struck by a halo WIMP, in a lowbackground experiment [3,4], and neutrino indirectdetection $(\nu \mathrm{ID})$ via observation of energetic neutrinos from annihilation of WIMPs that have been captured in the Sun (and/or Earth) $[5,6]$.

The DD rate is proportional to the local dark-matter density. The $\nu$ ID rate is proportional to the rate at which WIMPs annihilate in the Sun, which in turn depends on an integral of the square of the dark-matter density over the volume of the Sun. However, the WIMPs depleted in the Sun by annihilation are replenished by the capture of new WIMPs. In most cases where the $\nu$ ID signal is large enough to be detectable, the time scale for equilibration of capture and annihilation is small compared with the age of the Solar System. The $\nu$ ID rate is then also determined by the local dark-matter density. Since the capture rate is controlled by the same elastic-scattering process that occurs in $\mathrm{DD}$, the $\mathrm{DD}$ and $\nu \mathrm{ID}$ rates are roughly proportional [7].

In this Letter, we investigate the effects of Galactic substructure on this canonical scenario. Analytic arguments and numerical simulations suggest that realistic Galactic halos should have significant substructure, rem- nants of smaller halos produced in early stages of the structure-formation hierarchy (which may themselves house remnants of even smaller structures, and so on) [8]. Theoretical arguments suggest that the substructure may be scale invariant [9] with subhalos extending all the way down to sub-Earth-mass scales [10]. The local dark-matter density of different locations at similar Galactocentric radii in the Milky Way may thus differ by a few orders of magnitude. The analytic descriptions of substructure are rough, and the simulations are limited by finite resolution, and this motivates the pursuit of avenues toward empirically probing the existence of substructure.

The purpose of this Letter to show that measurements of the ratio of DD to $\nu$ ID rates can be used to test for Galactic substructure. If there is Galactic substructure, then the dark-matter density at the position of the Solar System may vary with time. There is a finite time lag between capture and annihilation, and so the current energeticneutrino flux may be determined not by the local darkmatter density, but rather by the density of dark matter along the past trajectory of the Solar System. The ratio for the $\nu \mathrm{ID} / \mathrm{DD}$ rate may thus differ from the canonical prediction. A departure from the canonical ratio would thus, if observed, provide information about Galactic substructure. Since the equilibration time scale in the Earth is generally different from that in the Sun, additional information might be provided by observation of energetic neutrinos from WIMP annihilation in the Earth.

To illustrate, we suppose the WIMP has a scalar coupling to nuclei, but the formalism can be easily generalized to spin-dependent WIMPs. Then the DD rate for a WIMP of mass $m_{\chi}$ from a target nucleus of mass $m_{i}$ is [1,7],

$$
\begin{aligned}
R_{\mathrm{DD}}^{\mathrm{sc}}= & 2.2 \times 10^{5} \mathrm{~kg}^{-1} \mathrm{yr}^{-1} \rho_{\chi, 0.3} \eta_{c}\left(m_{\chi}, m_{i}\right)\left(\frac{m_{\chi}}{100 \mathrm{GeV}}\right) \\
& \times\left(\frac{m_{i}}{100 \mathrm{GeV}}\right)\left(\frac{m_{i}}{m_{\chi}+m_{i}}\right)^{2} \sigma_{40}
\end{aligned}
$$

where $\rho_{\chi, 0.3}$ is the local dark-matter density in units of 
$0.3 \mathrm{GeV} \mathrm{cm}^{-3}$, and $\eta_{c}\left(m_{\chi}, m_{i}\right)$ (given in Ref. [4]) accounts for form-factor suppression. Here, $\sigma_{40}$ is the cross section for WIMP-nucleon scattering in units of $10^{-40} \mathrm{~cm}^{2}$.

The flux of upward muons induced in a neutrino telescope by neutrinos from WIMP annihilation in the Sun is

$$
\begin{aligned}
\Gamma_{\nu, 0}= & 7.3 \times 10^{5} \mathrm{~km}^{-2} \mathrm{yr}^{-1}\left(N / N_{\mathrm{eq}}\right)^{2} \rho_{\chi, 0.3} f\left(m_{\chi}\right) \\
& \times\left[\xi\left(m_{\chi}\right) / 0.1\right]\left(m_{\chi} / 100 \mathrm{GeV}\right)^{2} \sigma_{40},
\end{aligned}
$$

while the corresponding flux from Earth is obtained by replacing the prefactor of Eq. (2) by $15 \mathrm{~km}^{-2} \mathrm{yr}^{-1}$. The function $f\left(m_{\chi}\right)$ varies over the range $5 \leq f\left(m_{\chi}\right) \leq 0.5$ over the mass range $10 \leq m_{\chi} / \mathrm{GeV} \leq 1000$ for the Sun (with a slightly larger range for the Earth), while the function $\xi\left(m_{\chi}\right)$ is in the range $\sim 0.01-0.3$ over the same mass range.

The factor $N / N_{\text {eq }}$ in Eq. (2) quantifies the number of WIMPs in the Sun. Once WIMPs are captured in the Sun, they accumulate deep within the solar core, where they may annihilate to a variety of heavy Standard Model particles which then decay to produce high-energy neutrinos (which may escape the Sun). The time $t$ evolution of the number $N$ of WIMPs in the Sun is governed by the differential equation,

$$
d N / d t=C_{c}-C_{a} N^{2},
$$

where $C_{c}$ is the capture rate of WIMPs by the Sun, and $C_{a} N^{2}$ is twice (because each annihilation destroys two WIMPs) the effective annihilation rate. If both $C_{c}$ and $C_{a}$ are constant and the initial condition is $N(t=0) \equiv N_{0}$, the solution to this equation is

$$
N(t)=\sqrt{\frac{C_{c}}{C_{a}}} \frac{e^{t / \tau}-\gamma e^{-t / \tau}}{e^{t / \tau}+\gamma e^{-t / \tau}},
$$

where

$$
\gamma \equiv \frac{1-N_{0} \sqrt{C_{a} / C_{c}}}{1+N_{0} \sqrt{C_{a} / C_{c}}} \leq 1
$$

and $\tau=1 / \sqrt{C_{c} C_{a}}$ is the equilibration time scale. After a time $t \gtrsim \tau$, the number $N$ approaches $N_{\text {eq }} \equiv N(t \gg \tau)=$ $\sqrt{C_{c} / C_{a}}$, and the annihilation rate $\Gamma_{\nu}$ becomes equal to (one half) the capture rate, $\Gamma_{\nu}=C_{a} N^{2} / 2=C_{c} / 2$.

The capture rate $C_{c}$ and annihilation coefficient $C_{a}$, and thus the equilibration time scale $\tau$, are determined by the cross sections for WIMPs to annihilate and to scatter from nuclei. The equilibration time scale evaluates to

$$
\begin{aligned}
\tau_{\odot}= & 1.9 \times 10^{5} \operatorname{yrs}\left[\rho_{\chi, 0.3} f\left(m_{\chi}\right)\left(\sigma_{A} v\right)_{26}\right]^{-1 / 2} \\
& \times\left(m_{\chi} / 100 \mathrm{GeV}\right)^{-3 / 4} \sigma_{40}^{-1 / 2} .
\end{aligned}
$$

Here, $\left(\sigma_{A} v\right)_{26}$ is the annihilation cross section (times relative velocity $v$ in the limit $v \rightarrow 0$ ) in units of $10^{-26} \mathrm{~cm}^{-3} \mathrm{~s}^{-1}$. The equilibration time scale for the
Earth is obtained by replacing the prefactor of Eq. (6) by $1.1 \times 10^{8} \mathrm{yr}$. Using the canonical numbers we have adopted, the equilibration time scales for the Sun and Earth are both small compared with the age of the Solar System, but the equilibration time scales may vary by several orders of magnitude over reasonable ranges of the WIMP parameter space (and even more if more exotic physics, like a Sommerfeld [11] or self-capture enhancement [12], is introduced). To illustrate, we show in Fig. 1 the equilibration time scales, for various DD and $\nu$ ID rates, for realistic supersymmetric dark-matter candidates (using DARKSUSY [13]).

Suppose now the WIMP model parameters are determined, e.g., from the LHC and/or by theoretical assumption or modeling. Then, the unknowns in Eqs. (1) and (2) will be the halo density $\rho_{\chi}$ and the number $N$ of WIMPs in the Sun (or Earth). The measured DD rate will then provide the local halo density $\rho_{\chi}$. Measurement of the $\nu$ ID rate will then determine $\left(N / N_{\text {eq }}\right)=\tanh (t / \tau)$ (in both the Sun and the Earth).

For example, suppose the equilibration time scale is $\tau_{\odot} \approx 10^{7}$ years in the Sun, and that the Solar System entered a region of density $\rho=100 \rho_{\odot}$ (where $\rho_{\odot}=$ $0.3 \mathrm{GeV} \mathrm{cm}^{-3}$ is the smoothed local halo density) a time $\delta t \approx 10^{6}$ years ago, e.g., a $10^{9} M_{\odot}$ halo (see Fig. 2). We would then see a boosted DD rate and a boosted energeticneutrino flux from the Sun, but the energetic-neutrino flux

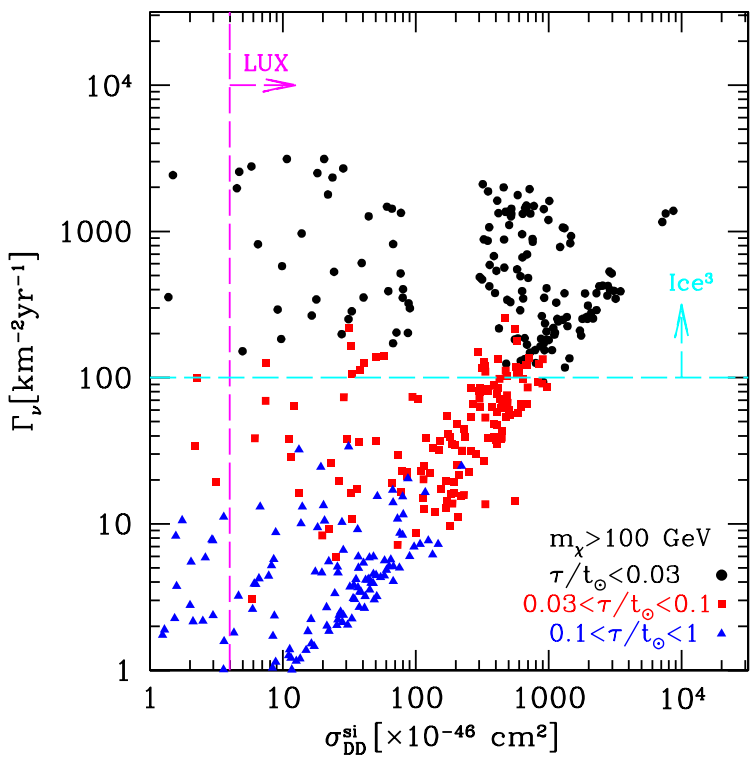

FIG. 1 (color online). The flux of energetic neutrinos from the Sun versus the rate for direct detection. Each point denotes a supersymmetric model with the correct relic density and consistent with experiment. The different symbols indicate the time scale for equilibration between capture and annihilation in the Sun. The horizontal line indicates a flux-threshold target for future $\nu$ ID experiments (IceCube + DeepCore) [15] and the vertical line a rate-threshold target for $\mathrm{DD}$ in a 3-ton liquidxenon detector [16]. For current bounds, see [17]. 


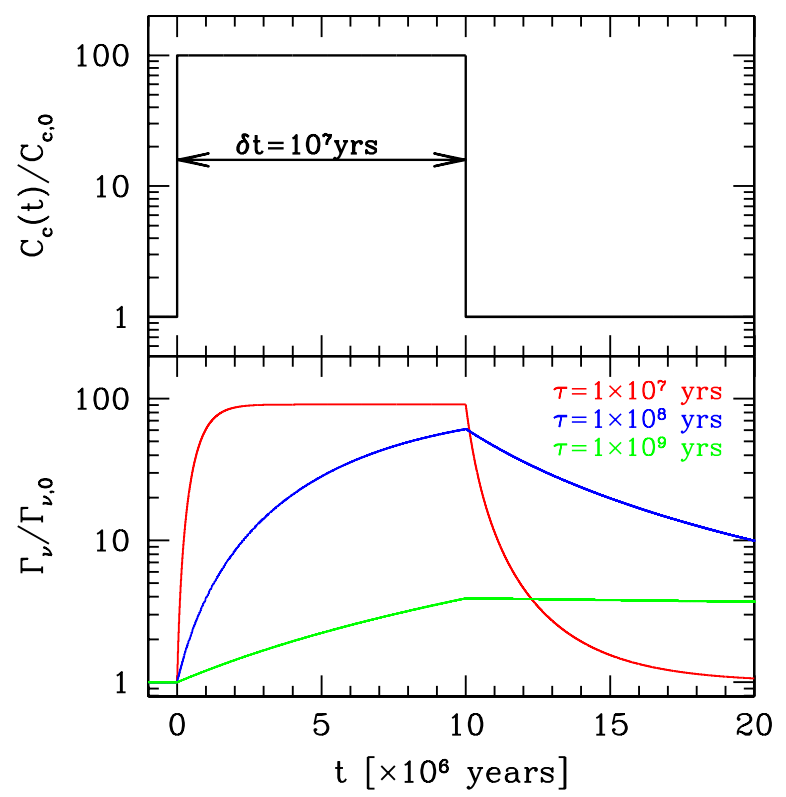

FIG. 2 (color online). The neutrino-flux enhancement from an encounter, of duration $10^{7} \mathrm{yr}$, of the Solar System with a region where the dark-matter density is enhanced by a factor of 100 (e.g., a $10^{9} M_{\odot}$ subhalo). The top panel shows the capture rate (i.e., the DD rate). The bottom panel shows the resulting energetic-neutrino flux from such an encounter for three equilibration time scales.

from the Earth would be correspondingly weaker, since the equilibration time in the Earth is longer. Now, suppose that the Solar System exited this high-density region a million years ago. The DD rate would be at the canonical value, but the energetic-neutrino fluxes from the Sun or Earth would still be boosted. Finally, suppose that the Solar System exited the high-density region $10^{7}$ years ago. In that case, the DD rate and energetic-neutrino flux from the Sun would have the canonical values, but the neutrino flux from the Earth would still be boosted, as $\tau_{\oplus}>\tau_{\odot}$.

In reality, the capture rate $C_{c}(t)$ in Eq. (3) is a function of time, and the equation for the number of WIMPs in the Sun or Earth can be integrated numerically to give the annihilation rate $C_{a} N^{2}(t) / 2$ as a function of time. To illustrate, imagine that all of halo dark matter was distributed in objects of a single mass, $M=M_{1} M_{\odot}$, each with a density $1000 \beta \rho_{\odot}[14]$. The radius of these subhalos would then be $R=0.1 \mathrm{pc}\left(M_{1} / \beta\right)^{1 / 3}$. The transit time of the Solar System through such an object is $\delta t \approx 1000\left(M_{1} / \beta\right)^{1 / 3} \mathrm{yr}$. The mean-free time between encounters with such objects is $\bar{t} \approx 10^{7}\left(M_{1} \beta^{2}\right)^{1 / 3}$ yr. In this toy model, the dark-matter density (and hence the DD rate) is zero unless the Solar System is within a subhalo. Figure 3 illustrates the effects from such a scenario for $M_{1}=1$. For equilibration time scales which are of order $\tau \sim \bar{t}$, the energetic-neutrino signal is depleted completely prior to the next encounter, while for longer equilibration time scales, the net effect is an elevated signal at all times. For example, if $\tau_{\odot} \sim \bar{t}$, and

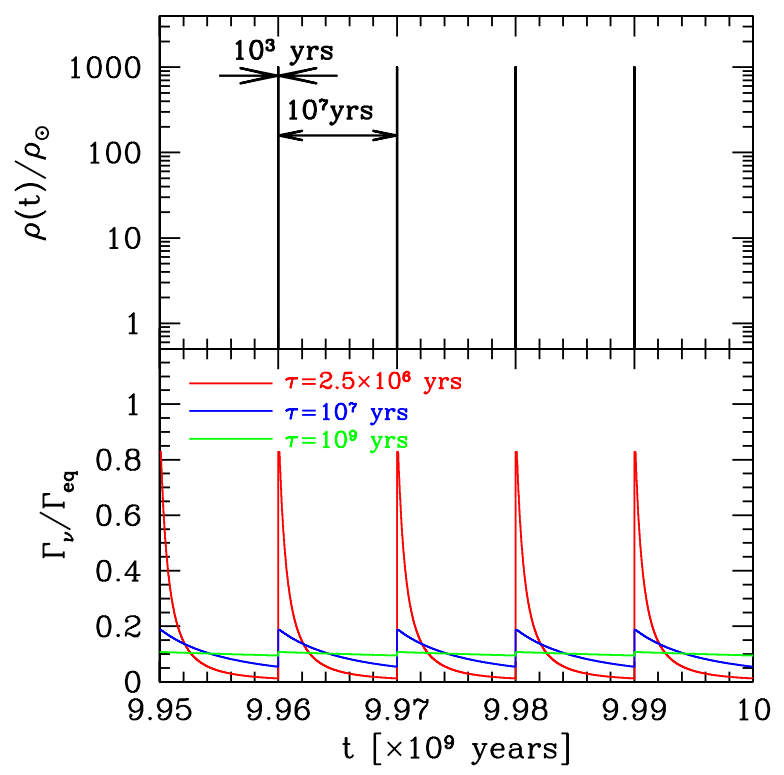

FIG. 3 (color online). The neutrino flux in a hypothetical scenario where all dark matter is in dense objects of $1 M_{\odot}$. Different curves correspond to equilibration time scales as shown. Short equilibration time scales (e.g., Sun) almost deplete completely the amount of WIMPs in a time comparable to the time scale between interactions. Longer equilibration time scales (e.g., Earth) result in a constant elevated flux.

$\tau_{\oplus}>\tau_{\odot}$, the signal from the Earth will be boosted relative to the signal from the Sun for most of the time.

Finally, substructure may also speed up the equilibration between capture and annihilation in cases where the smooth-halo equilibration time scale is larger than the age of the Solar System. Suppose the dark matter has a smooth component and some substructure down to very small scales, $M \sim 10^{-6} M_{\odot}$. The abundance of the smallest subhalos can be inferred by extrapolating the subhalo mass function measured in simulations at larger mass scales. If we take the density within these $10^{-6} M_{\odot}$ objects to be $\sim 100$ times the smooth value, the radius of these objects is $10^{-2} \mathrm{pc}$, the crossing time is 50 years, and the mean time between encounters is roughly a million years. For equilibration time scales less than the age of the Sun $\left(\tau_{\odot} \ll t_{\odot}\right)$, the signal will be roughly at the equilibrium value of the smooth component for most of the time. However, for long equilibration time scales (e.g., the Earth), the amount of depletion between interactions is negligible. This effect leads to a continuous buildup of WIMPs in the Earth, augmented by brief periods of an increased capture due to interactions with the subhalos. This results in an energetic-neutrino signal that today is higher than the signal that would be obtained from the smooth component. This can be understood as follows: For $N \ll N_{\text {eq }}$, the second term in Eq. (3) is small. While the cross section for the Solar System trajectory to intersect subhalos is $\propto \beta^{-2 / 3}$, the capture rate while in them is $\propto \beta$, thus giving 


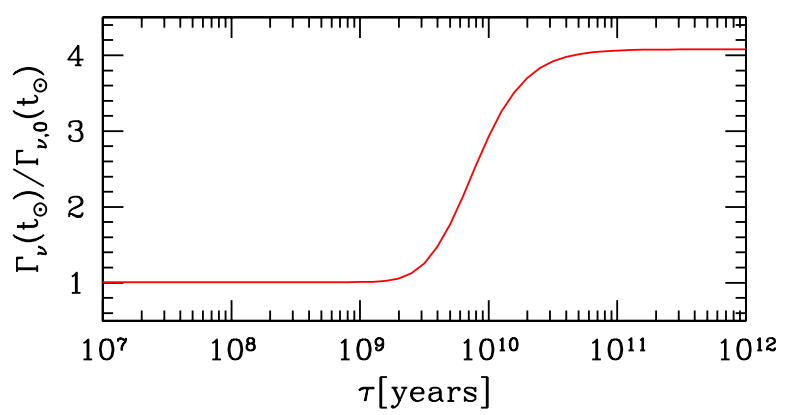

FIG. 4 (color online). The net effect on the energetic-neutrino flux of the presence of $10^{-6} M_{\odot}$ objects along the solar Galactic radius during the whole lifetime of the Solar System. Long equilibration time scales result in the net buildup of WIMPs and thus an increase in the neutrino flux relative to that which would be obtained in a smooth halo.

rise to a net increase in the capture rate $\propto \beta^{1 / 3}$. Figure 4 shows the net effect of this speedup.

In summary, we considered the effects of Galactic substructure on energetic neutrinos from WIMP annihilation in the Sun and the Earth. While DD experiments depend on the local dark-matter density and velocity distribution, the energetic-neutrino fluxes from the Sun and the Earth depend on the past trajectory of the Solar System through the clumpy Galactic halo. If experimental DD and $\nu$ ID signals are obtained before the dark-matter particle-physics parameters are known, then the potential for probing dark matter via the $\mathrm{DD} / \nu \mathrm{ID}$ ratios will be compromised by the particle-physics uncertainties. If, however, the particlephysics parameters are known, then measurement of the DD rate and the $\nu$ ID rates from the Sun and Earth can be used to probe Galactic substructure at the solar radius, with the equilibration time scales setting roughly the mass scales that can be probed.

We acknowledge useful conversations with J. Beacom, I. Dell'Antonio, R. Gaitskell, A. Geringer-Sameth, G. Jungman, L. Strigari, and A. Zentner. S. M. K. thanks the Caltech/JPL W. M. Keck Institute for Space Studies for hospitality during the preparation of this Letter. The work of M.K. was supported by DoE No. DE-FG03-92ER40701 and the Gordon and Betty Moore Foundation.

*koushiappas@brown.edu

†kamion@tapir.caltech.edu

[1] G. Jungman, M. Kamionkowski, and K. Griest, Phys. Rep. 267, 195 (1996); L. Bergstrom, Rep. Prog. Phys. 63, 793 (2000); G. Bertone, D. Hooper, and J. Silk, Phys. Rep. 405, 279 (2005).

[2] H. C. Cheng, J. L. Feng, and K. T. Matchev, Phys. Rev. Lett. 89, 211301 (2002); G. Servant and T. M.P. Tait, Nucl. Phys. B650, 391 (2003); For a review, see D. Hooper and S. Profumo, Phys. Rep. 453, 29 (2007).
[3] M.W. Goodman and E. Witten, Phys. Rev. D 31, 3059 (1985); I. Wasserman, Phys. Rev. D 33, 2071 (1986).

[4] K. Griest, Phys. Rev. D 38, 2357 (1988); 39, 3802(E) (1989).

[5] J. Silk, K. A. Olive, and M. Srednicki, Phys. Rev. Lett. 55, 257 (1985); W. H. Press and D. N. Spergel, Astrophys. J. 296, 679 (1985); L. M. Krauss, K. Freese, W. Press, and D. Spergel, Astrophys. J. 299, 1001 (1985); T. K. Gaisser, G. Steigman, and S. Tilav, Phys. Rev. D 34, 2206 (1986); L. M. Krauss, M. Srednicki, and F. Wilczek, Phys. Rev. D 33, 2079 (1986); K. Freese, Phys. Lett. B 167, 295 (1986); A. Gould, Astrophys. J. 328, 919 (1988); 388, 338 (1992); M. Kamionkowski, Phys. Rev. D 44, 3021 (1991); F. Halzen, T. Stelzer, and M. Kamionkowski, Phys. Rev. D 45, 4439 (1992); G. Jungman and M. Kamionkowski, Phys. Rev. D 51, 328 (1995).

[6] A. Gould, Astrophys. J. 321, 571 (1987).

[7] M. Kamionkowski et al., Phys. Rev. Lett. 74, 5174 (1995).

[8] S. Ghigna et al., Mon. Not. R. Astron. Soc. 300, 146 (1998); A. A. Klypin et al., Astrophys. J. 522, 82 (1999); A. A. Klypin, S. Gottlober, and A. V. Kravtsov, Astrophys. J. 516, 530 (1999); B. Moore et al., Astrophys. J. 524, L19 (1999); V. Berezinsky, V. Dokuchaev, and Y. Eroshenko, Phys. Rev. D 77, 083519 (2008); J. Diemand, M. Kuhlen, and P. Madau, Astrophys. J. 667, 859 (2007); C. Giocoli, L. Pieri, and G. Tormen, arXiv:0712.1476; A. Helmi, S.D.M. White, and V. Springel, Phys. Rev. D 66, 063502 (2002); D. Reed et al., Mon. Not. R. Astron. Soc. 359, 1537 (2005); V. Springel et al., arXiv:0809.0898.

[9] M. Kamionkowski and S. M. Koushiappas, Phys. Rev. D 77, 103509 (2008).

[10] A. M. Green, S. Hofmann, and D. J. Schwarz, Mon. Not. R. Astron. Soc. 353, L23 (2004); J. Cosmol. Astropart. Phys. 08 (2005) 003; A. Loeb and M. Zaldarriaga, Phys. Rev. D 71, 103520 (2005); E. Bertschinger, Phys. Rev. D 74, 063509 (2006); J. Diemand, M. Kuhlen, and P. Madau, Astrophys. J. 649, 1 (2006); J. Diemand, B. Moore, and J. Stadel, Nature (London) 433, 389 (2005); H. Zhao et al., Astrophys. J. 654, 697 (2007); H. S. Zhao et al., arXiv: astro-ph/0502049; T. Goerdt et al., Mon. Not. R. Astron. Soc. 375, 191 (2007); V. Berezinsky, V. Dokuchaev, and Y. Eroshenko, Phys. Rev. D 73, 063504 (2006); X.1. Chen, M. Kamionkowski, and X.m. Zhang, Phys. Rev. D 64, 021302 (2001); S. Profumo, K. Sigurdson, and M. Kamionkowski, Phys. Rev. Lett. 97, 031301 (2006).

[11] J. Hisano, S. Matsumoto, M. M. Nojiri, and O. Saito, Phys. Rev. D 71, 063528 (2005).

[12] A. R. Zentner, arXiv:0907.3448 [Phys. Rev. D (to be published)].

[13] P. Gondolo, J. Edsjo, P. Ullio, L. Bergstrom, M. Schelke, and E. A. Baltz, J. Cosmol. Astropart. Phys. 07 (2004) 008.

[14] S. M. Koushiappas, arXiv:0905.1998; J. Diemand, P. Madau, and B. Moore, Mon. Not. R. Astron. Soc. 364, 367 (2005); T. Bringmann, arXiv:0903.0189.

[15] T. DeYoung, Mod. Phys. Lett. A 24, 1543 (2009).

[16] http://luxdarkmatter.org.

[17] Z. Ahmed et al. (CDMS Collaboration), Phys. Rev. Lett. 102, 011301 (2009); J. Angle et al., Phys. Rev. Lett. 101, 091301 (2008); S. Desai et al., Phys. Rev. D 70, 083523 (2004); J. Braun, H. Daan (IceCube Collaboration), arXiv:0906.1615. 